\section{THE PROBLEM OF AN ULTRA-NEPTUNIAN} PLANET.

$\mathrm{I}^{\mathrm{N}}$ this memoir ${ }^{1}$ Prof. W. H. Pickering first gives a graphical method by which Neptune might have been discovered from the data used by Le Verrier and Adams. Having thus demonstrated the practical value of his method, he proceeds to apply it to a search for a planet beyond Neptune. He calls his hypothetical planet by the letter " $O$," obviously as the next letter of the alphabet to the initial of Neptune, and he finds, inter alia,

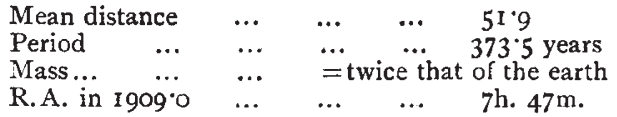

Now the problem presented by Uranus, Neptune, and " $O$ " may be very readily reduced to the known problem, already fully worked out, of Mercury, Venus, and the earth, for it is easy to show that the theory of a pair of planets is the same if we retain the masses but alter the distances of both in the same proportion.

First of all, as, roughly speaking, we are only able to observe heliocentric longitudes of Uranus and Neptune, we must suppose that our fictitious observer, to whom the existence of the earth is unknown, is only able to observe heliocentric longitudes of Mercury and Venus. We may speak of him, therefore, as an observer in the sun.

Secondly, as the mass of " $\mathrm{O}$ " is twice that of the earth, we must credit the observer in the sun with instruments of twice the precision of those used by ourselves.

Lastly, we may divide all distances by $5 \mathrm{I}$, and all time intervals by 365 . This latter factor enables us to substitute days for years.

We are, therefore, to suppose that an observer in the sun, with instruments of twice the accuracy of our own, has observed Mercury for four months and Venus for two months, and that in addition he has one or two stray observations of Mercury and Venus made before he recognised its planetary character.

Now let us turn to the tables of Mercury and Venus, and estimate for ourselves what chance such an observer has of demonstrating the existence of the earth.

Before doing this, however, we must pause for a moment in order to show that we may dismiss from notice all long-period terms. These terms play a very conspicuous part in planetary theory. Their existence depends upon the same principle as that of the swing, where a very small force applied at regular and suitable intervals will produce very large oscillations. Their existence necessitates the expansion of the disturbing function to ten or a hundred times the accuracy otherwise necessary, and the consequence is that from the computer's point of view the shortperiod terms are dismissed with scanty notice in the account that he gives of his work.

For example, in the heliocentric longitude of Venus there is a term

$$
3^{\prime \prime} \sin (\mathrm{r} 3 \mathrm{E}-8 \mathrm{~V})
$$

with a period of 239 years. Our hypothetical observer in the sun would have no chance of detecting such a term as this. If he detected in two months' observations any term at all it would be one of the following :-

$$
\begin{gathered}
5^{\prime \prime} \sin (V-E) \\
I r^{\prime \prime} \sin 2(V-E) \\
7^{\prime \prime} \sin 3(V-E)
\end{gathered}
$$

the periods being 585,292 , and 195 days respectively.

1 "Annals of the Astronomical Observatory of Harvard College." Vol. Ixi., part ii. A Search for a Planet beyond Nept une. By W. H. Pickering. (Cambridge, Mass. : The Observatory, Ig09.)
It may further be observed that it is the long-period terms which are largely affected by small changes in the elements of the disturbing planet; the shortperiod terms are not appreciably affected. During the hypothetical two months of observation the question is, "Where is the disturbing planet at that time?" not "Are the elements such as produce a long-period term?"

Further, it is not sufficient that a term of moderate amplitude and period should exist. In the hypothetical two months, only a very small portion of a complete period is observed, and the conditions must be such that the term is not mistaken for uniform motion or for a term periodic in the period of the planet under observation, for in either case the term could be represented by a change of the elliptic elements of the orbit.

At this point we wish to say that we do not think Prof. Pickering's case is a good one, and, having thus proclaimed ourselves adverse critics, we wish immediately to concede the following point. In our opinion the hypothetical observer could detect from two months' observations the term $7^{\prime \prime} \sin 3(\mathrm{~V}-\mathrm{E})$ in the heliocentric longitude of Venus, and could distinguish it from a mere error of assumed elliptic elements, provided only the phase of the argument happened to be suitable during the period of observation. Revert. ing now to the actual case, the existence of planet "O " could be demonstrated from the observations of Neptune at the present date if the epoch of planet " $O$ " were suitable. If the observations of Neptune show nothing, the hypothesis of the existence of " $O$ " would not be negatived, but the hypothetical " $\mathrm{O}$ " would at least be confined to certain limits of longitude.

Prof. Pickering, however, has based his discovery of " $O$," not on Neptune, but on Uranus. In the hypothetical case we have to consider the perturbations of Mercury by the earth. In this case we have terms such as

$$
\begin{aligned}
& 0 \cdot 2^{\prime \prime} \sin (M-E) \\
& 0^{\circ} 3^{\prime \prime} \sin 2(M-E),
\end{aligned}
$$

which we believe to be quite incapable of detection from four months' observations, or at any rate of detection and distinction from elliptic terms. In saying this we do not forget that the terms must be doubled to take account of the double mass attributed to "O."

It will be seen that our criticisms are directed against Prof. Pickering's figures as we find them. We have argued against the extremely small mass assigned to the hypothetical planet, seeing that Prof. Piclsering's data are the observations of Uranus. While constructing our argument we have, however, convinced ourselves that the time is ripe for a discussion of the observations of Neptune, for if the planet " $\mathrm{O}$ " exists, or any approximation to it, it should have produced, or at any rate should scon produce, a visible effect on Neptune.

\section{NOTES.}

MeEtings of two special commissions appointed by the International Meteorological Committee at Paris in 1907 will be held in London during the week commencing Monday next, June $2 \mathrm{r}$. The appointment of the first commission arose out of a proposal made at Innsbruck by the Rev. Lowis Froc, S.J., director of the Zi-ka-wei Observatory, for the general adoption of a code of maritime weather signals now in use in far eastern waters, and a further proposal made at Paris by Prof. Willis Moore, chief of the United States Weather Bureau, in favour of an international system of maritime weather signals. To 